\title{
General intelligence and modality-specific differences in performance: a response to Schellenberg (2008)
}

\author{
ADAM T. TIERNEY \\ Department of Cognitive Science, UC San Diego \\ TONYA R. BERGESON \\ Department of Otolaryngology, Indiana University School of Medicine \\ DAVID B. PISONI \\ Department of Psychological and Brain Science, Indiana University
}

\begin{abstract}
Tierney et al. (2008) reported that musicians performed better on an auditory sequence memory task when compared to non-musicians, but the two groups did not differ in performance on a sequential visuo-spatial memory task. Schellenberg (2008) claims that these results can be attributed entirely to differences in IQ. This explanation, however, cannot account for the fact that the musicians' advantage was modality-specific.
\end{abstract}

Submitted 2009 February 17; accepted 2009 February 18.

KEYWORDS: memory span, musical training, general aptitude, music performance, skilled musicians, sequence learning

BECOMING proficient at music involves the development of an extremely wide variety of perceptual and motor skills. Expert keyboardists, for example, need to be able to, without looking, suddenly move their hand two or three feet away and strike with one finger an area less than half an inch wide-not just once but many times in rapid succession. They must have memorized hours of music, they must be able to, in real time, translate music notation into both auditory imagery and motor commands, and they must be able to hear a complex array of sounds, separate out its component instruments, and follow the pitch and rhythm of each.

It is an open question to what extent these abilities transfer into non-musical life, giving rise to more general aptitudes. If they do, one might expect trained musicians to enjoy benefits ranging from more efficient visual encoding strategies (necessary for comprehending oncoming groups of notes at a glance while sight-reading), to longer auditory memory spans, to enhanced fine-motor control. Since these simple, low-level abilities contribute to many more complicated tasks, it would not be surprising to find that musicians enjoy a small advantage in complex domains such as mathematics and verbal reasoning as well, which likely benefit from more efficient visual encoding and higher auditory memory capacity, respectively. Further complicating the picture is the fact that not all musicians undergo the same sorts of training-some musicians are never trained to play by ear, and many never become proficient at sight-reading (or, in fact, learn to read music at all). Moreover, different instruments require very different sets of cognitive, perceptual, and motor skills.

In fact, there is growing evidence that taking music lessons is associated with proficiency in a wide variety of tasks, including mathematics (Hetland, 2000), spatial skills (Vaugh, 2000), verbal memory (Chan, Ho, \& Cheung, 1998; Ho, Cheung, \& Chan, 2003; Jakobson, Lewycky, Kilgour, \& Stoesz, 2008) and IQ (Schellenberg, 2004, 2006), among others. It is tempting to respond to this complicated picture with a simple explanation: taking music lessons is linked to higher "general intelligence", either as a consequence of the musical training, or because children with higher IQ are more likely to end up playing an instrument (Schellenber 2008). This explanation only works, however, if one is willing to grant "general intelligence" existence, and explanatory power, separate from any perceptual, motor, or cognitive skills that may comprise it. For if IQ tests merely examine a wideranging set of abilities, claiming that increases in, say, visuo-spatial memory span are actually due to higher IQ amounts to saying that one's math grades got better because one's G.P.A. increased.

An alternative explanation is that music performance is a complex activity with auditory, visual, somatosensory, and motor components, and that the wide variety of skills that have been linked 
to music practice and instruction are simply a reflection of this diversity. If this view is correct, it should be possible to find well-defined cognitive or perceptual tasks that are not linked to music proficiency. One possible candidate is sequential visuo-spatial memory, or memory for sequences of locations in space. Highly trained musicians have been found (e.g., Lee, Lu, \& Ko, 2007; Jakobson, Lewycky, Kilgour, \& Stoesz, 2008) to have greater visual memory spans, but these tests have all involved memorization of the details of single complex objects or sequences of such objects, rather than sequences of locations in space.

We hypothesized that highly trained classical pianists would display increased performance on tests of auditory-verbal memory, due to their having memorized extensive passages of music, but not on tests of sequential visuo-spatial memory. To test this hypothesis, we measured performance of skilled musicians from the Indiana University School of Music on a test of verbal and visuo-spatial memory span. For both the verbal and visuo-spatial memory span tests, subjects were asked to remember and press sequences of buttons on a "Simon" memory box, which contains four buttons of different colors arranged in a circle. During the verbal test, subjects heard and attempted to memorize sequences of color names, then reproduced them by pressing the buttons immediately after. During the visuo-spatial test, the buttons lit up in sequence, and the subjects were asked to remember and reproduce the sequence by pressing the buttons. The output and, in an abstract sense, the information to be remembered in both of these tests was identical-the only difference being the modality in which the information was presented. In addition to the trained musicians, we also tested a variety of comparison groups-trained gymnasts who began studying at an early age, psychology 101 students, and students who habitually played video games.

The musicians significantly outperformed all three comparison groups on the auditory condition. No difference was found between the groups on the visual condition. Differences in IQ cannot, as Schellenberg (2008) claims, account for this effect, because differences in general intelligence should not give rise to disparities in performance on only one of two such similar tests. Schellenberg offers no explanation for how differences in IQ could give rise to this result.

As our paper admitted freely, because we did not use a truly experimental design, we could "at best only uncover non-causal relations between musical experience and non-musical cognitive skills". Although a plausible explanation of our results is that musicians' training facilitates auditory sequence memory, an alternative account is that only those musicians proficient in the memorization of auditory sequences are drawn to music as a career or are able to gain admission to the Indiana University School of Music. A possible follow-up study could attempt to establish causality and further investigate which components of music lessons are linked to auditory-verbal memory span by giving children music lessons in which ear training and memorization either are or are not heavily emphasized. We hypothesize that music lessons not containing these crucial components would not lead to facilitated auditory-verbal memory.

\section{REFERENCES}

Chan, S., Ho, Y. \& Cheung, M. (1998). Music training improves verbal memory. Nature, Vol. 396, 128 .

Ho, Y.C., Cheung, M.C. \& Chan, A.S. (2003) Music training improves verbal but not visual memory: Cross-sectional and longitudinal explorations in children. Neuropsychology, Vol. 17, 439-450.

Jakobson, L.S., Lewycky, S.T., Kilgour, A.R. \& Stoesz, B.M. (2008). Memory for verbal and visual material in highly trained musicians. Music Perception, Vol. 26, 41-55.

Lee, Y., Lu, M. \& Ko, H. (2007). Effects of skill training on working memory capacity. Learning and Instruction, Vol. 17, 336-344.

Schellenberg, E.G. (2004). Music lessons enhance IQ. Psychological Science, Vol. 15, 511-514.

Schellenberg, E.G. (2006). Long-term positive associations between music lessons and IQ. Journal of Educational Psychology, Vol. 98, 457-468. 
Schellenberg, E.G. (2008). Commentary on "'Effects of early musical experience of auditory sequence memory" by Adam Tierney, Tonya Bergeson, and David Pisoni', Empirical Musicology Review, Vol. 3, 205-207.

Stoesz, B.M., Jakobson, L.J., Kilgour, A.R. \& Lewycky, S.T. (2007). Local processing advantage in musicians: Evidence from disembedding and constructional tasks. Music Perception, Vol. 25, 153-165.

Tierney, A., Bergeson, T. \& Pisoni, D. (2008). Effects of early musical experience on auditory sequence memory, Empirical Musicology Review, Vol. 3, 178-186.

Vaughn, K. (2000). Music and mathematics: Modest support for the oft-claimed relationship. Journal of Aesthetic Education, Vol. 34, No. 3/4, 149-166. 